\title{
Rectification of Fluctuations in an Underdamped Ratchet
}

\author{
Ya. M. Blanter and M. Büttiker \\ Département de Physique Théorique, Université de Genève, CH-1211, Genève 4, Switzerland
}

(May 12, 2018)

\begin{abstract}
We investigate analytically the motion of underdamped particles subject to a deterministic periodic potential and a periodic temperature. Despite the fact that an underamped particle experiences the temperature oscillation many times in its escape out of a well and in its motion along the potential, a net directed current linear in the friction constant is found. If both the potential and the temperature modulation are sinusoidal with a phase lag $\delta$, this current is proportional to $\sin \delta$.
\end{abstract}

PACS numbers: $05.40 .+\mathrm{j}, 82.20 . \mathrm{Mj}, 87.10 .+\mathrm{e}$

Recently, research on molecular motors [1,2], which apparently operate with an efficiency close to $k T$, has stimulated interest in ratchets. Ratchets are commonly defined as systems which, in the absence of a net force or macroscopic gradient, are able to produce a directed current through the rectification of noise [3]. Fundamentally ratchets are of interest as simple non-equilibrium systems. Some of the proposed models of ratchets consider particles in a periodic potential and subject to a non-uniform periodic temperature profile 田目, or subject to a time-dependent external force with zero average [8], which, in particular, can be stochastic. Other models invoke Brownian motion in fluctuating potentials [9] or models of Brownian particles which can be in several states with external pumping of particles to one of the states [10]. These works treat overdamped particles. Models in which inertia plays a role either within classical or quantum dynamics have been considered only recently 11, 12, and the results available by now are mostly numerical.

We present analytical results for the motion of underdamped particles in a periodic potential and subject to a periodic temperature modulation (see Fig. 1). In the particular case shown in Fig. 1a, both the temperature modulation and the potential are sinusoidal with the same microscopic period and a relative phase lag $\delta$. We show that a directed current results which is proportional to $\sin \delta$. No current is observed if the system is symmetric $\delta=n \pi$. This is the underdamped analog of a similar effect which occurs for overdamped motion in systems with state dependent diffusion [1 6 . In the overdamped case, directed motion appears because a particle diffusing up a potential hill can easier surmount a potential barrier if the ascent occurs in a heated region [13. In contrast, in the presence of a very small frictional force, a particle experiences the microscopic temperature modulation many times, both when the particle is in a well and even after its escape into a running state. Thus, the current must vanish in the zero dissipation limit.

The motion of a classical particle in an external potential and subject to thermal noise is governed by Kramers' equation [14]. While the overdamped motion (Smolu- chowski limit) is relatively easy to handle, the underdamped limit is much more complex. The steady state
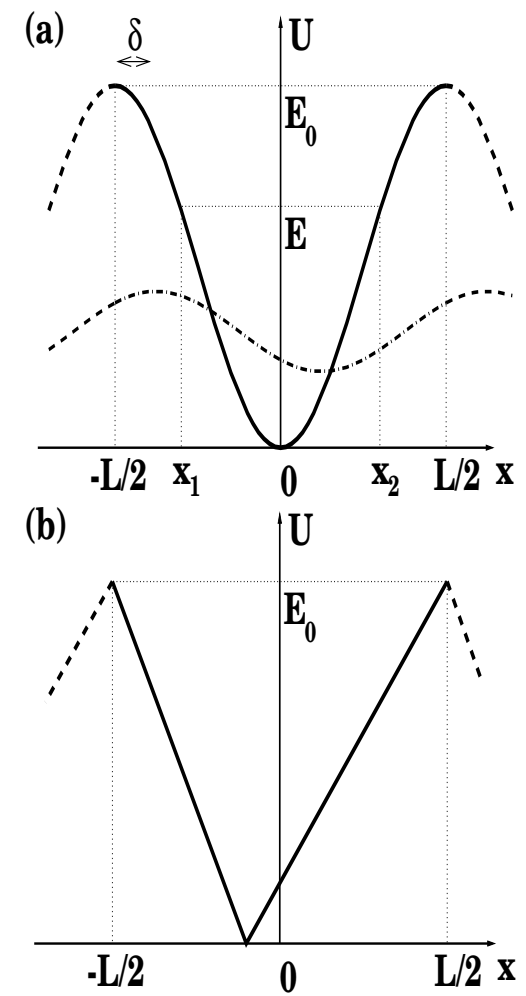

FIG. 1. One period of the potential $U(x)$, (a) the potential given by Eq.(17) (bold solid line), temperature variation $T(x)$ (dash-dotted line); (b) an example of sawtooth potential.

current of particles in a periodic potential due to a constant external force was treated by Risken and Vollmer [15]. Here we generalize their approach to the case of a spatially non-uniform temperature, thus presenting a rare example of a solution of an underdamped problem. Kramers' equation [14] for the joint distribution function $W(x, v)$ of coordinate and velocity for a particle with mass $m$ in the potential $U(x)$ subject to a frictional force with damping constant $\gamma$ and a temperature $T(x)$ is 


$$
-m v \frac{\partial W}{\partial x}+\frac{\partial U}{\partial x} \frac{\partial W}{\partial v}+\gamma \frac{\partial}{\partial v}\left[\frac{T(x)}{m} \frac{\partial W}{\partial v}+v W\right]=0 .
$$

We assume that both $U(x)$ and $T(x)$ are periodic functions with the same period $L$, and the potential $U(x)$ has only one maximum per oscillation period. The amplitude of the potential is $E_{0}$, the zero of energy is chosen at the minima of the potential, and the points $x= \pm L / 2$ correspond to the potential maxima (Fig. 1). Furthermore we assume that $T(x) \ll E_{0}$ for any $x$.

Eq. (11) must be supplemented by boundary conditions. For $E>E_{0}$ the distribution function is periodic in $x, W(-L / 2, v)=W(L / 2, v)$. For energies below $E_{0}$ all particles are reflected from the potential barrier at the points $x_{1}(E)$ and $x_{2}(E)$ (Fig. 1). Thus, for $E<E_{0}$, the boundary condition requires that at the turning points the distribution function of incoming particles is the same as that of the reflected particles.

Following Ref. [15], we replace the velocity $v$ by the energy variable $E=m v^{2} / 2+U(x)$. It is then necessary to discriminate between left- and right-moving particles; their distribution functions are denoted as $W^{-}$ and $W^{+}$, respectively. Introducing for further convenience the symmetric and antisymmetric combinations, $W^{S, A}=W^{+} \pm W^{-}$, we obtain 15

$$
\begin{aligned}
& \frac{\partial W^{A}}{\partial x}=\gamma \frac{\partial}{\partial E}\left\{v(x, E)\left[1+T(x) \frac{\partial}{\partial E}\right] W^{S}(x, E)\right\} ; \\
& \frac{\partial W^{S}}{\partial x}=\gamma \frac{\partial}{\partial E}\left\{v(x, E)\left[1+T(x) \frac{\partial}{\partial E}\right] W^{A}(x, E)\right\},
\end{aligned}
$$

with the boundary conditions

$$
\begin{array}{cc}
W^{S, A}(-L / 2, E)=W^{S, A}(L / 2, E), & E>E_{0} \\
W^{A}\left(x_{1}(E), E\right)=W^{A}\left(x_{2}(E), E\right)=0, & E<E_{0}
\end{array} .
$$

Here $v(x, E)=[(2 / m)(E-U(x))]^{1 / 2}$, and the turning points $x_{1,2}(E)$ are the solutions of the equation $U(x)=$ $E$. In addition, both functions $W^{S}$ and $W^{A}$ and their energy derivatives are continuous for $E=E_{0}$. The function $W^{S}$ is normalized according to

$$
\int d E d x[m v(x, E)]^{-1} W^{S}(x, E)=1,
$$

and the expression for the (particle) current has the form

$$
I=m^{-1} \int d E W^{A}(x, E) .
$$

Integrating Eq. (2) over energy, we conclude immediately that the current $I$ does not depend on $x$. Note that no assumption about damping has been made so far equations (2), (3) are valid for arbitrary $\gamma$.

Now we turn to the underdamped case. For $\gamma=0$, both the symmetric and the antisymmetric distribution functions $W^{S}$ and $W^{A}$ depend on energy only. Then due to the boundary conditions $W^{A}$ vanishes for $E<E_{0}$, and the matching conditions for $E=E_{0}$ imply that it vanishes also for $E>E_{0}$. Thus in the absence of friction there is no current [16]. Dissipation is essential for the migration of particles between different potential wells. For low dissipation the typical time for this migration is much longer than the period of the oscillations inside each well, and thus the energy varies much slower in time than the coordinate $x$ of the particle. Consequently, the distribution function depends weakly on $x$ while it varies rapidly with energy. This rapid dependence is, however, essential only in the narrow layer of energies around $E=E_{0}$; the thickness of this layer is proportional to a positive power of $\gamma$. Generalizing the approach of Ref. [15] we write

$$
W^{S, A}(x, E)=\tilde{W}^{S, A}(x, E)+w^{S, A}(x, E),
$$

where $\tilde{W}^{S, A}$ are slow functions of $x$ and $E$, while $w^{S, A}(x, E)$ are slow functions of $x$ that vary rapidly with energy. The functions $w^{S, A}$ are different from zero only in the narrow layer of energies around $E_{0}$; their role is to ensure the continuity of $W^{S, A}$ for $E=E_{0}$. For $E>E_{0}$, the boundary conditions read now

$$
\begin{gathered}
\tilde{W}^{S, A}(-L / 2, E)=\tilde{W}^{S, A}(L / 2, E) ; \\
w^{S, A}(-L / 2, E)=w^{S, A}(L / 2, E),
\end{gathered}
$$

and for $E<E_{0}$

$$
\tilde{W}^{A}\left(x_{1,2}(E), E\right)=w^{A}\left(x_{1,2}(E), E\right)=0 .
$$

First we consider the functions $\tilde{W}^{S, A}(x, E)$. The periodic functions $v(x, E), t(x, E) \equiv v(x, E) T(x)$ and the distributions $\tilde{W}^{S, A}(x, E)$ can be expanded in Fourier series (in the range $E<U(x)$ we define them to be zero),

$$
\begin{gathered}
v(x, E)=v_{0}+\sum_{n=1}^{\infty} v_{n c} \cos \frac{2 \pi n x}{L}+\sum_{n=1}^{\infty} v_{n s} \sin \frac{2 \pi n x}{L} \\
t(x, E)=t_{0}+\sum_{n=1}^{\infty} t_{n c} \cos \frac{2 \pi n x}{L}+\sum_{n=1}^{\infty} t_{n s} \sin \frac{2 \pi n x}{L} ; \\
\tilde{W}^{S, A}(x, E)=\tilde{W}_{0}^{S, A}(E)+\sum_{n=1}^{\infty} \tilde{W}_{n c}^{S, A}(E) \cos \frac{2 \pi n x}{L} \\
+\sum_{n=1}^{\infty} \tilde{W}_{n s}^{S, A}(E) \sin \frac{2 \pi n x}{L} .
\end{gathered}
$$

It is seen from Eqs. (2), (3) that the coefficients $\tilde{W}_{n c, s}^{A}$ are proportional to $\gamma$. Then for $E<E_{0}$ due to the boundary condition the whole function $\tilde{W}^{A}$ is proportional to $\gamma$, and consequently the $x$-dependent part of $\tilde{W}^{S}$ is proportional to $\gamma^{2}$. In the following we restrict our consideration to the terms proportional to $\gamma$, thus neglecting the coefficients $\tilde{W}_{n c, s}^{S}$. Taking the constant part of Eq. (2), we find for $E<E_{0}$ 


$$
\tilde{W}^{S}(E) \equiv N P(E)=N \exp \left\{-\int_{E_{0}}^{E} \frac{v_{0}\left(E^{\prime}\right)}{t_{0}\left(E^{\prime}\right)} d E^{\prime}\right\},
$$

with a normalizing constant $N$. With the abbreviation $\phi(x, E)=1-\left(v_{0}(E) / t_{0}(E)\right) T(x)$ the solution for $\tilde{W}^{A}$ satisfying the boundary condition for $E<E_{0}$ is

$$
\tilde{W}^{A}(x, E)=\gamma \frac{\partial}{\partial E}\left\{\tilde{W}^{S}(E) \int_{x_{1}(E)}^{x} v\left(x^{\prime}, E\right) \phi\left(x^{\prime}, E\right) d x^{\prime}\right\} .
$$

We note that for potentials $U(x)$ (such as that of Fig. 1a) with a saddle at $E_{0}$, this expression diverges as $E \rightarrow E_{0}$ and $x \rightarrow \pm L / 2$. This divergence is similar to that of the oscillation period for $E \rightarrow E_{0}$. It is not detrimental, since the Jacobian from $(x, v)$ to the $(x, E)$ space has a compensating singularity. It is advantageous to treat this divergence by considering a sawtooth potential, like that of Fig. 1b, for which all expression converge. We will proceed in this way, and our final results will be shown to be convergent in general case. Thus, we expect that our consideration is also valid for potentials with a saddle.

For $E>E_{0}$ the function $\tilde{W}^{S}$ still does not depend on $x$ (up to terms of order $\gamma$ ), and is given by Eq. (10). For $\tilde{W}^{A}(x, E)$ we find

$$
\begin{aligned}
\tilde{W}^{A}(x, E) & =\gamma \frac{\partial}{\partial E}\left\{\tilde{W}^{S}(E) \int_{-L / 2}^{x} v\left(x^{\prime}, E\right) \phi\left(x^{\prime}, E\right) d x^{\prime}\right\} \\
& +B(E),
\end{aligned}
$$

which differs from the solution for $E<E_{0}$ by the position-independent function $B(E)$. The latter appears due to the different boundary conditions, and is determined by Eq. (3). Taking the constant part of this equation and fixing the only integration constant so that $B\left(E_{0}\right)=0$, we obtain

$$
B(E)=P(E)\left[\int_{E_{0}}^{E} \frac{F\left(E^{\prime}\right)}{P\left(E^{\prime}\right)} d E^{\prime}+G\left(E_{0}\right)\right]-G(E) .
$$

Here we have introduced the functions

$$
\begin{aligned}
& F(E)=-\frac{1}{2 t_{0}(E)} \sum_{n=1}^{\infty} \sum_{i=c, s}\left[v_{n i}(E) \tilde{W}_{n i}^{A}+t_{n i}(E) \frac{\partial \tilde{W}_{n i}^{A}}{\partial E}\right], \\
& G(E)=-\frac{\gamma}{L} \frac{\partial}{\partial E}\left\{\tilde{W}^{S}(E) \int_{-L / 2}^{L / 2} x v(x, E) \phi(x, E) d x\right\} .
\end{aligned}
$$

The functions $\tilde{W}_{n c, s}^{A}$ (which are just the coefficients in the Fourier series for the solution Eq. (11)) are easily obtained from Eq. (2) and read

$$
\tilde{W}_{n c, s}^{A}= \pm \frac{\gamma L}{2 \pi n} \frac{\partial}{\partial E}\left\{\left[v_{n s, c}(E)+t_{n s, c}(E) \frac{\partial}{\partial E}\right] \tilde{W}^{S}(E)\right\} .
$$

With the help of the antisymmetric product,

$$
\{a \times b\}=\left(2 L^{2}\right)^{-1} \int_{-L / 2}^{L / 2} a(x) b\left(x^{\prime}\right) \operatorname{sign}\left(x-x^{\prime}\right) d x d x^{\prime},
$$

the expression for $B(E)$ after some algebra can be brought into the form

$$
B(E)=-\gamma L N P(E) \int_{E_{0}}^{E} \frac{d E^{\prime}}{t_{0}\left(E^{\prime}\right)}\left[\left\{v \times \frac{\partial v}{\partial E^{\prime}}\right\}-\frac{v_{0}}{t_{0}}\left\{v \times \frac{\partial t}{\partial E^{\prime}}\right\}-2 \frac{v_{0}}{t_{0}}\left\{t \times \frac{\partial v}{\partial E^{\prime}}\right\}+2 \frac{v_{0}^{2}}{t_{0}^{2}}\left\{t \times \frac{\partial t}{\partial E^{\prime}}\right\}\right] .
$$

Now we cure the discontinuity of $\partial \tilde{W}^{A} / \partial E$ for $E=E_{0}$ by taking into account the functions $w^{S, A}(x, E)$. Since they vary rapidly with energy, the derivatives $\partial w / \partial E$ can be eliminated in favor of $\partial^{2} w / \partial E^{2}$. Replacing $E$ by $E_{0}$ in the functions which are smooth in the energy, neglecting the difference between $x_{1}(E)$ and $-L / 2$, and introducing the dimensionless variable $u$,

$$
u(x)=-\pi+2 \pi \frac{\int_{-L / 2}^{x} T(x) v\left(x, E_{0}\right) d x}{\int_{-L / 2}^{L / 2} T(x) v\left(x, E_{0}\right) d x}
$$

(which is an analog of the action for the uniform temperature case), $-\pi \leq u \leq \pi$, we find

$$
\frac{\partial w^{S, A}}{\partial u}=u_{0} \frac{\partial^{2} w^{A, S}}{\partial E^{2}}
$$

Here $u_{0}=\gamma L t_{0}\left(E_{0}\right) /(2 \pi)$, and the boundary conditions read $w^{A}( \pm \pi, E)=0$ for $E<E_{0}$, and $w^{S, A}(-\pi, E)=$ $w^{S, A}(\pi, E)$ for $E>E_{0}$.
Eq. (14) has been investigated by Risken and Vollmer 15. Making use of their solution, writing down the matching conditions for $W^{A}$ at $E=E_{0}$, and taking into account that the function $B(E)$ in Eq. (11) is proportional to $\gamma$, we find that the functions $w^{S, A}$ are proportional to $\gamma^{3 / 2}$. Thus, these functions are not essential for our consideration. For the current we obtain then

$$
I=m^{-1} \int_{E_{0}}^{\infty} B(E) d E .
$$

Eqs. (13) and (15) together with the definition (5) of the normalizing constant $N$ yield a closed expression for the current induced by an arbitrary temperature distribution $T(x)$. This current is proportional to $\gamma$ and vanishes in the limit of constant temperature $T(x)=T_{0}$, since then $t(x, E)=T_{0} v(x, E)$.

We continue with two simplifying assumptions. First, we consider the experimentally relevant regime of small temperature gradients. Specifically, following Ref. [4], we 
assume

$$
T(x)=T_{0}+T_{1} \cos (2 \pi x / L-\delta), \quad T_{1} \ll T_{0} .
$$

Furthermore, for simplicity, we choose the potential $U(x)$ to be symmetric, $U(x)=U(-x)$. To linear order in $T_{1}$, the expression for the current simplifies and is given by

$$
\begin{aligned}
I & =\frac{\gamma L N T_{1}}{m T_{0} L^{2} v_{0}\left(E_{0}\right)} \sin \delta \int_{-L / 2}^{L / 2} d x \sin \left(\frac{2 \pi x}{L}\right) \frac{\partial v\left(x, E_{0}\right)}{\partial E} \\
& \times \int_{0}^{x} v\left(x^{\prime}, E_{0}\right) d x^{\prime}
\end{aligned}
$$

where we used the condition $T_{0} \ll E_{0}$. As is expected, the current is proportional to $\sin \delta$, and vanishes when both the potential and the temperature are symmetric. We also note that Eq. (16) is finite for a potential $U(x)$ of a general form (even though $\partial v\left(x, E_{0}\right) / \partial x$ diverges for $x \rightarrow \pm L / 2$ ), and thus, we expect that it is generally valid. As an example, we consider the simple form of $U(x)$,

$$
U(x)=\left(E_{0} / 2\right)[1-\cos (2 \pi x / L)],
$$

shown in Fig. 1a. We obtain

$$
I=\frac{\gamma T_{1}}{2 m T_{0}} \exp \left(-\frac{E_{0}}{T_{0}}\right) \sin \delta .
$$

It is instructive to compare this expression with the one found in the overdamped regime [4.5] (index ov). For the same parameters we find

$$
I_{o v}=\frac{\pi^{2} E_{0}^{2} T_{1}}{\gamma T_{0}^{2} L^{2}} \exp \left(-\frac{E_{0}}{T_{0}}\right) \sin \delta .
$$

First, we note that the sign of the current is the same in the overdamped and in the underdamped regime. It corresponds to the notion [13,4 that it is easier for the particle to climb a "hot" slope. Furthermore, we note that the two expressions (18) and (19) match for a certain critical damping,

$$
\gamma_{c} \sim\left(E_{0} / L\right)\left(m / T_{0}\right)^{1 / 2}
$$

This critical damping can be understood by considering the overdamped side. Indeed, to leading order, the overdamped approximation [4, neglects the terms which do not contain $\gamma$ in Eq. (11). This is justified for $\gamma \gg v^{-2} \partial U / \partial x$, where $v \sim(T / m)^{1 / 2}$ is the typical thermal velocity. Substituting $E_{0} / L$ for the characteristic value of the potential derivative, we readily obtain Eq. (20). Thus, we conclude that our expression for the underdamped regime (18) is valid up to $\gamma \sim \gamma_{c}$.

Now we compare the result (18) with the current $I \sim(F / \gamma L) \exp \left(-E_{0} / T_{0}\right)$, obtained in Ref. 15 for the underdamped Brownian motion due to an external force $F$. We see that a non-uniform temperature has the same effect as an applied effective uniform driving force
$F_{\text {eff }} \sim \gamma^{2} L T_{1} \sin \delta /\left(m T_{0}\right)$. Thus, the motion generated by a non-uniform temperature profile is less effective than the forced motion due to a uniform field. This is in contrast to the overdamped case [4], where the effective force $F_{\text {eff }} \sim E_{0} T_{1} \sin \delta /\left(T_{0} L\right)$ does not depend on $\gamma$.

In conclusion, we investigated how a non-uniform temperature can produce a steady, directed current of particles in a periodic potential in the underdamped regime. A current is generated even in the case of a symmetric potential provided the temperature profile is asymmetric (out of phase). A temperature oscillation is equivalent to an external driving force with a magnitude proportional to the square of the friction constant.

The work was supported by the Swiss National Science Foundation.

[1] F. Jülicher, A. Ajdari and J. Prost, Rev. Mod. Phys. 69, 1269 (1997); R. D. Astumian, Science, 276, 917 (1997).

[2] R. D. Vale and F. Oosawa, Adv. Biophys., Ed. by M. Kotani (Elsevier, Limerick, 1990) 26, 97; A. Ajdari and J. Prost, C. R. Acad. Sci. Paris II 315, 1635 (1992).

[3] R. P. Feynman, R. B. Leighton and M. Sands, The Feynman Lectures in Physics (Addison-Wesley, Reading, 1966), Vol. 1, Chap. 46.

[4] M. Büttiker, Z. Phys. B 68, 161 (1987).

[5] N. G. van Kampen, IBM J. Res. Dev. 32, 107 (1988).

[6] R. Landauer, J. Stat. Phys. 53, 233 (1988).

[7] A model equivalent to the application of spatial dependent temperature was derived from the microscopic classical description of a system coupled to a bath of oscillators, see M. M. Millonas, Phys. Rev. Lett. 74, 10 (1995); ibid, 75, 3027E (1995); A. M. Jayannavar, Phys. Rev. E 53, 2957 (1996).

[8] M. O. Magnasco, Phys. Rev. Lett. 71, 1477 (1993); R. Bartussek, P. Hänggi, and J. G. Kissner, Europhys. Lett. 28, 459 (1994); M. M. Millonas and M. I. Dykman, Phys. Lett. A 185, 65 (1994); C. R. Doering, W. Horsthemke, and J. Riordan, Phys. Rev. Lett. 72, 2984 (1994); I. Derényi and T. Vicsek, ibid, 75, 374 (1995).

[9] R. D. Astumian and M. Bier, Phys. Rev. Lett. 72, 1766 (1994).

[10] J. Prost, J.-F. Chauwin, L. Peliti, and A. Ajdari, Phys. Rev. Lett. 72, 2652 (1994).

[11] P. Jung, J. Kissner, and P. Hänggi, Phys. Rev. Lett. 76, 3436 (1996).

[12] S. Yukawa, M. Kikuchi, G. Tatara, and H. Matsukawa, J. Phys. Soc. Jap. 66, 2953 (1997); P. Reinmann, M. Grifoni, and P. Hänggi, Phys. Rev. Lett. 79, 10 (1997); G. Tatara, M. Kikuchi, S. Yukawa, and H. Matsukawa, cond-mat/9711045 (unpublished); I. A. Goychuk, E. G. Petrov, and V. May, Phys. Lett A 238, 59 (1998). 
[13] Bistable systems with state dependent diffusion have long been of interest to demonstrate that in non-equilibrium systems the relative stability of locally stable states is affected by the kinetics even far from these states. See R. Landauer, Phys. Rev. A 12, 636 (1975).

[14] H. A. Kramers, Physica 7, 284 (1940).
[15] H. Risken and H. D. Vollmer, Z. Phys. B 35, 177 (1979).

[16] This is in contrast to the system driven by a constant force, see e.g. [15]. This force breaks the symmetry between $v$ and $-v$, implying an infinite current for vanishing dissipation. 